\title{
Percutaneous parametrial dose escalation in women with advanced cervical cancer: feasibility and efficacy in relation to long-term quality of life
}

\author{
Sati Akbaba',2, Jan Tobias Oelmann-Avendano, ${ }^{1,2}$, Tilman Bostel ${ }^{1,2}$, Harald Rief ${ }^{1,2,3}$, \\ Nils Henrik Nicolay ${ }^{1,2,4,5}$, Juergen Debus ${ }^{1,2,4}$, Katja Lindel ${ }^{1,2,6}$, Robert Foerster ${ }^{1,2,7}$ \\ 1 Department of Radiation Oncology, University Hospital Heidelberg, Heidelberg, Germany \\ 2 National Center for Radiation Research in Oncology (NCRO), Heidelberg Institute for Radiation Oncology (HIRO), \\ Heidelberg, Germany \\ ${ }^{3}$ Gemeinschaftspraxis Strahlentherapie Bonn-Rhein-Sieg, Bonn, Germany \\ ${ }^{4}$ Clinical Cooperation Unit Radiation Oncology, German Cancer Research Center (DKFZ), Heidelberg, Germany \\ ${ }^{5}$ Department of Radiation Oncology, University Hospital Freiburg, Freiburg, Germany \\ ${ }^{6}$ Department of Radiation Oncology, Staedtisches Klinikum Karlsruhe, Karlsruhe, Germany \\ 7 Department of Radiation Oncology, University Hospital Zurich, Zurich, Switzerland
}

Radiol Oncol 2018; 52(3): 320-328.

Received 14 February 2018

Accepted 09 May 2018

Correspondence to: Robert Foerster, M.D., Department of Radiation Oncology, University Hospital Zurich, Raemistrasse 100,8091 Zurich, Switzerland. Phone: +41 44255 9959; Fax: +41 44255 4547; E-mail: robert.foerster@usz.ch

Disclosure: No potential conflict of interest were disclosed.

Background. We analyzed long-term quality of life (QOL) and prognostic factors for QoL as well as clinical outcome in patients with advanced cervical cancer (ACC) treated with primary radiochemotherapy (RChT) consisting of external beam radiotherapy (EBRT) with or without sequential or simultaneous integrated boost (SIB) to the parametria, intracavitary brachytherapy and concomitant chemotherapy (ChT).

Patients and methods. Eighty-three women were treated with primary RChT between 2008 and 2014. Survival of all patients was calculated and prognostic factors for survival were assessed in univariate and multivariate analysis. In 31 patients QoL was assessed in median 3 years (range 2-8 years) after treatment. QoL was compared to published normative data and the influence of age, tumour stage, treatment and observed acute toxicities was analyzed.

Results. Thirty-six patients (43.4\%) died, 18 (21.7\%) had a local recurrence and 24 (28.9\%) had a distant progression. Parametrial boost $(p=0.027)$ and $C h T(p=0.041)$ were independent prognostic factors for overall survival in multivariate analysis. Specifically, a parametrial equivalent doses in 2-Gy fractions (EQD2) $>50$ Gy was associated with an improved overall survival (OS) $(p=0.020)$, but an $E Q D 2>53$ Gy did not further improve $O S(p=0.194)$. Tumour size was the only independent prognostic factor for local control $(p=0.034)$. Lymph node status $(p=0.038)$ and distant metastases other than in paraaortic lymph nodes $(p=0.002)$ were independent prognostic factors for distant progressionfree survival. QoL was generally inferior to the reference population. Age only correlated with menopausal symptoms $(p=0.003)$. The degree of acute gastrointestinal $(p=0.038)$ and genitourinary $(p=0.041)$ toxicities correlated with the extent of chronic symptom experience. Sexual/vaginal functioning was reduced in patients with larger tumours $(p=0.012)$. Parametrial EQD2 > 53 Gy correlated with reduced sexual/vaginal functioning $(p=0.009)$ and increased sexual worry $(p=0.009)$. Whether parametrial dose escalation was achieved by sequential boost or SIB, did not affect survival or QoL.

Conclusions. Primary RChT is an effective treatment, but long-term QoL is reduced. The degree of acute side effects of RChT correlates with the extent of chronic symptoms. Patients benefit from parametrial SIB or sequential boost, but an EQD2 > 53 Gy does not further improve survival and negatively affects QoL.

Key words: cervical cancer; parametrial boost; quality of life; radiotherapy 


\section{Introduction}

Primary radiochemotherapy (RChT) with external beam radiotherapy (EBRT), intracavitary brachytherapy (ICBT), and concomitant chemotherapy (ChT) remains a frequently used treatment for advanced cervical cancer (ACC). However, local tumour control requires comparatively large doses, which in turn may lead to relevant treatmentrelated morbidity. ${ }^{1,2}$ In recent years, image guided adaptive brachytherapy (IGABT), based on magnetic resonance imaging (MRI) and combined interstitial / intracavitary brachytherapy (IBT/ICBT), has been successfully implemented as the new standard of care for local dose escalation and substantial reduction of therapy-related morbidity. ${ }^{3-5}$

However, access to IGABT is limited at many brachytherapy facilities. Therefore, despite evidence of the inferiority of percutaneous boosting in terms of organ sparing and target coverage, another approach has been to combine ICBT with modern radiotherapy (RT) techniques, such as intensity-modulated radiotherapy (IMRT) with simultaneous integrated boost (SIB) to the parametria, for percutaneous local dose escalation..$^{6,7}$ There is a low incidence of acute toxicities after SIB, but clinical outcome and QoL of these patients have not been investigated so far. ${ }^{7}$ Due to limited access to IGABT at many brachytherapy facilities and the unknown effects of SIB in terms of chronic morbidity, IMRT with sequential boost remains a widely used method for parametrial dose escalation. Given the comparatively young age of patients with cervical cancer and improving prognosis, long-term quality of life (QoL) and extent of chronic morbidity become increasingly important issues.

Therefore, we conducted this study to analyze feasibility and efficacy of a percutaneous parametrial boost in relation to long-term QoL as well as to assess tumour- and treatment-related prognostic factors for long-term QoL and outcome in women with ACC.

\section{Patients and methods}

Between 2008 and 2014, eighty-three women with ACC underwent primary treatment at our department. Patients' data were acquired from the institutional electronic patient charts and the institutional follow-up database. Median age at first diagnosis was 57 years (range 32-90 years; Table 1). Due to the substantially differing fractionation schedules, all reported doses were recalculated as equivalent
TABLE 1. Patients' characteristics

\begin{tabular}{|c|c|c|}
\hline \multicolumn{3}{|l|}{ Age } \\
\hline Median & \multicolumn{2}{|c|}{57 years } \\
\hline \multirow[t]{2}{*}{ Range } & \multicolumn{2}{|c|}{$32-90$ years } \\
\hline & $n$ & $\%$ \\
\hline \multicolumn{3}{|l|}{ Histology } \\
\hline Squamous cell carcinoma & 67 & $80.7 \%$ \\
\hline Adenocarcinoma & 14 & $16.9 \%$ \\
\hline Adenosquamous carcinoma & 2 & $2.4 \%$ \\
\hline \multicolumn{3}{|l|}{ Grading } \\
\hline G1 & 8 & $9.6 \%$ \\
\hline G2 & 24 & $28.9 \%$ \\
\hline G3 & 35 & $42.2 \%$ \\
\hline GX & 16 & $19.3 \%$ \\
\hline \multicolumn{3}{|l|}{ FIGO stage } \\
\hline 1 & 2 & $2.4 \%$ \\
\hline$\|$ & 40 & $48.2 \%$ \\
\hline III & 15 & $18.1 \%$ \\
\hline IV & 26 & $31.3 \%$ \\
\hline \multicolumn{3}{|l|}{ Tumour size } \\
\hline $\mathrm{T} 1$ & 3 & $3.6 \%$ \\
\hline $\mathrm{T} 2$ & 46 & $55.4 \%$ \\
\hline T3 & 25 & $30.1 \%$ \\
\hline $\mathrm{T} 4$ & 9 & $10.8 \%$ \\
\hline \multicolumn{3}{|l|}{ Lymph node status } \\
\hline NO & 28 & $33.7 \%$ \\
\hline $\mathrm{NI}$ & 55 & $66.3 \%$ \\
\hline \multicolumn{3}{|l|}{ Distant metastases } \\
\hline MO & 64 & $77.1 \%$ \\
\hline Mla & 12 & $14.5 \%$ \\
\hline Mlc & 7 & $8.4 \%$ \\
\hline
\end{tabular}

doses in 2-Gy fractions with $\alpha / \beta=10$ (EQD2 ${ }_{10}$ ) for the tumour and $\alpha / \beta=3\left(\mathrm{EQD} 2_{3}\right)$ for the organs at risk (OARs). OAR doses were documented for volumes of $0.1 \mathrm{~cm}^{3}$ (D0.1cc), $1 \mathrm{~cm}^{3}$ (D1cc) and $2 \mathrm{~cm}^{3}$ (D2cc). They were calculated as the sum of the individual doses received from all brachytherapy fractions and the EBRT plan. ${ }^{8}$

Survival was plotted according to Kaplan and Meier. Overall survival (OS) was defined as the time between first diagnosis and death. Local progression-free survival (LPFS) was defined as the time between first diagnosis and occurrence of any local progression. Since patients with distant metastases (cM1a and cM1c) at first diagnosis were in- 
TABLE 2. Treatment and toxicity

\begin{tabular}{|c|c|c|}
\hline \multicolumn{3}{|c|}{ Radiotherapy dose in EQD2 $(\alpha / \beta=10)$} \\
\hline Median EBRT & \multicolumn{2}{|c|}{$44 \mathrm{~Gy}$} \\
\hline Range EBRT & \multicolumn{2}{|c|}{ 35-51 Gy } \\
\hline Median parametria & \multicolumn{2}{|c|}{$53 \mathrm{~Gy}$} \\
\hline Range parametria & \multicolumn{2}{|c|}{ 38-67 Gy } \\
\hline Median HDR-BT & \multicolumn{2}{|c|}{$40 \mathrm{~Gy}$} \\
\hline \multirow[t]{2}{*}{ Range HDR-BT } & \multicolumn{2}{|c|}{ 10-50 Gy } \\
\hline & $n$ & $\%$ \\
\hline \multicolumn{3}{|c|}{ Radiotherapy technique } \\
\hline IMRT & 58 & $69.9 \%$ \\
\hline 3D-conformal & 25 & $30.1 \%$ \\
\hline \multicolumn{3}{|l|}{ Parametrial boost } \\
\hline No boost & 36 & $43.4 \%$ \\
\hline Sequential & 31 & $37.3 \%$ \\
\hline SIB & 16 & $19.3 \%$ \\
\hline \multicolumn{3}{|c|}{ Parametrial dose in EQD2 $(\alpha / \beta=10)$} \\
\hline$\leq 53 \mathrm{~Gy}$ & 56 & $67.5 \%$ \\
\hline$>53 \mathrm{~Gy}$ & 27 & $32.5 \%$ \\
\hline \multicolumn{3}{|c|}{ Simultanous chemotherapy } \\
\hline Cisplatin 40 mg/m² & 71 & $85.5 \%$ \\
\hline None & 12 & $14.5 \%$ \\
\hline \multicolumn{3}{|l|}{ Total treatment duration } \\
\hline$<6$ weeks & 9 & $10.8 \%$ \\
\hline 6-8 weeks & 52 & $62.7 \%$ \\
\hline$\geq 9$ weeks & 22 & $26.5 \%$ \\
\hline \multicolumn{3}{|l|}{ Anemia during therapy } \\
\hline $\min . \mathrm{Hb}<10 \mathrm{~g} / \mathrm{dl}$ & 38 & $45.8 \%$ \\
\hline $\min . \mathrm{Hb} \geq 10 \mathrm{~g} / \mathrm{dl}$ & 45 & $54.2 \%$ \\
\hline \multicolumn{3}{|l|}{ Transfusions } \\
\hline$\leq 2 \mathrm{ECs}$ & 69 & $83.1 \%$ \\
\hline$>2 \mathrm{ECs}$ & 14 & $16.9 \%$ \\
\hline \multicolumn{3}{|c|}{ Observed acute GI toxicity } \\
\hline Grade 0 & 33 & $39.8 \%$ \\
\hline Grade I & 15 & $18.1 \%$ \\
\hline Grade ॥ & 20 & $24.1 \%$ \\
\hline Grade III & 12 & $14.5 \%$ \\
\hline Grade IV & 3 & $3.6 \%$ \\
\hline \multicolumn{3}{|c|}{ Observed acute GU toxicity } \\
\hline Grade 0 & 15 & $18.1 \%$ \\
\hline Grade I & 34 & $41.0 \%$ \\
\hline Grade ॥ & 28 & $33.7 \%$ \\
\hline Grade III & 6 & $7.2 \%$ \\
\hline
\end{tabular}

$\mathrm{EBRT}=$ external beam radiotherapy; $\mathrm{ECs}=$ erythrocyte concentrates; $\mathrm{EQD2}=$ equivalent doses in 2-Gy fractions; $\mathrm{Gl}=$ gastrointestinal; $\mathrm{GU}=$ genitourinary; $\mathrm{Hb}=$ haemoglobin; $\mathrm{HDR}-\mathrm{BT}$ = high-doserate brachytherapy; IMRT = intensity-modulated radiotherapy; $\mathrm{SIB}=$ simultaneous integrated boost cluded in this study, distant progression-free survival (DPFS) was defined as the interval between first diagnosis of cervical cancer and occurrence of new distant metastases. Prognostic factors for survival were analyzed with the log-rank test (univariate analysis) and a Cox proportional hazards model (multivariate analysis).

Three years (median; range 2-8 years) after treatment, patients were approached during clinical follow-up examinations and asked to fillin the European Organization for Research and Treatment of Cancer (EORTC) Quality of Life Questionnaire for Cancer Patients 30 (QLQ-C30) and the Cervical Cancer Module (QLQ-CX24). Thirty-one women agreed to participate. The difference between patients' QoL items scores and published German reference values was analyzed with the t-test (9). Possible prognostic factors for QoL (age, stage, tumour size, lymph node status, distant metastases status, histological grading, histology, RT techniques, applied RT doses, OAR doses, ChT, treatment duration, observed acute toxicities, anemia during RChT, number of transfusions during RChT) were investigated with an analysis of variances and the t-test. Age was used as a covariate. A p-value $\leq 0.05$ was considered statistically significant. All statistical analyses were performed with IBM SPSS version 24.0.

This study was conducted in accordance with the declaration of Helsinki and was approved by the responsible independent ethics committee on 22 October 2012 (\#S-513/2012). The requirement of informed consent was waived by the ethics committee, due to the retrospective nature of the study.

\section{Results}

\section{Treatment and dose-volume histogram analysis}

RT was conducted as EBRT (69.9\% IMRT, 30.1\% 3D-conformal RT), with or without sequential boost / SIB to parametria and involved pelvic / paraaortic lymph nodes (43.4\% no boost, $37.3 \%$ sequential boost, $19.3 \% \mathrm{SIB})$, and high-dose-rate (HDR) ICBT boost (tandem and ring applicator). Median EBRT EQD2 10 to the whole pelvic (and paraaortic) planning target volume (PTV) was $44 \mathrm{~Gy}$ (range 35-51 Gy) and median parametrial EQD2 ${ }_{10}$ was 53 Gy (range 38-67 Gy). Including ICBT boost, as prescribed to point $\mathrm{A}$, with a median EQD2 10 of $40 \mathrm{~Gy}$ (range 10-50 Gy), the median prescribed primary tumour EQD2 ${ }_{10}$ was 84 Gy (range 54-95 Gy). Seventy-one patients (85.5\%) received con- 
comitant ChT with 4-6 cycles of cisplatin $40 \mathrm{mg} /$ $\mathrm{m}^{2}$ weekly (Table 2 ).

Mean bladder D0.1cc, D1cc and D2cc were 130.2 Gy ( \pm SD 31.7 Gy), 105.6 Gy ( \pm SD 14.2 Gy) and 97.1 Gy ( \pm SD 9.9 Gy), respectively. Mean sigmoid D0.1cc, D1cc and D2cc were 71.9 Gy ( \pm SD 10.6 Gy), 64.1 Gy ( \pm SD 7.9 Gy) and 61.1 Gy ( \pm SD 6.9 Gy), respectively. Mean rectum D0.1cc, D1cc and D2cc were 89.8 Gy ( \pm SD 23.1 Gy), 74.3 Gy ( \pm SD 12.8 Gy) and $68.8 \mathrm{~Gy}( \pm$ SD $9.4 \mathrm{~Gy})$, respectively.

\section{Survival analysis}

Thirty-six patients $(43.4 \%)$ died, 18 (21.7\%) had a local progression and $24(28.9 \%)$ developed new distant metastases during follow-up. Calculated 3- / 5-year LPFS, DPFS and OS were 80.5\% / 73.2\%, $76.7 \% / 65.3 \%$ and $66.5 \% / 53.2 \%$, respectively.

In univariate analysis (Table 3), Fédération Internationale de Gynécologie et d'Obstétrique (FIGO) stage (I/II vs. III/IV; $\mathrm{p}=0.015)$, tumour size (T1/2 vs. T3/4; $\mathrm{p}=0.036$ ), parametrial boost (yes vs. no; $\mathrm{p}=0.037)$, parametrial dose $\left(\mathrm{EQD} 2_{10} \leq 50 \mathrm{~Gy} v\right.$ s. $>50$ Gy; $\mathrm{p}=0.020$ and EQD2 ${ }_{10}<53$ Gy vs. 53 Gy vs. $>53 \mathrm{~Gy} ; \mathrm{p}=0.017$ ), simultaneous ChT (yes vs. no; $\mathrm{p}$ $=0.004)$, total treatment duration (6-8 weeks vs. $\geq 9$ weeks; $\mathrm{p}=0.027$ ), anemia during therapy (minimal hemoglobin $<10 \mathrm{~g} / \mathrm{dl}$ vs. $\geq 10 \mathrm{~g} / \mathrm{dl} ; \mathrm{p}=0.039$ ) and number of erythrocyte concentrate transfusions $(\leq$ 2 vs. $>2 ; p=0.007$ ) were prognostic factors for OS. Histological grading showed a tendency towards statistical significance (G1/2 vs. G3; $\mathrm{p}=0.053)$. Importantly, a percutaneous dose escalation in the parametria beyond an EQD2 $2_{10}$ of 53 Gy did not further improve survival $(p=0.194$; Figure 1$)$. For DPFS, only tumour size $(\mathrm{T} 1 / 2$ vs. $\mathrm{T} 3 / 4 ; \mathrm{p}=0.034)$ was a prognostic factor. Lower FIGO stage (FIGO I/ II vs. III/IV; $\mathrm{p}=0.072$ ) and conductance of a parametrial boost (yes vs. no; $\mathrm{p}=0.095$ ) showed a tendency towards improved LPFS. DPFS was prolonged in patients without lymph node metastases (N0 vs. $\mathrm{N} 1 ; \mathrm{p}=0.038)$ and in patients without distant metastases other than in paraaortic lymph nodes (M0/ M1a vs. M1c; $p=0.002$ ) as well as in patients with low or intermediate histological grading (G1/2 vs. $\mathrm{G} 3 ; \mathrm{p}=0.037$ ). In none of three endpoints we found any difference in outcome between patients receiving SIB or sequential boost.

In multivariate analysis (Table 3), parametrial boost (yes vs. no, HR 0.417 [95\%CI 0.192-0.900], $\mathrm{p}=0.027$ ) and simultaneous ChT (yes vs. no, HR 0.382 [95\%CI 0.152-0.961], $\mathrm{p}=0.041$ ) remained as independent prognostic factors for OS. Advanced tumour size was the only independent prognos-

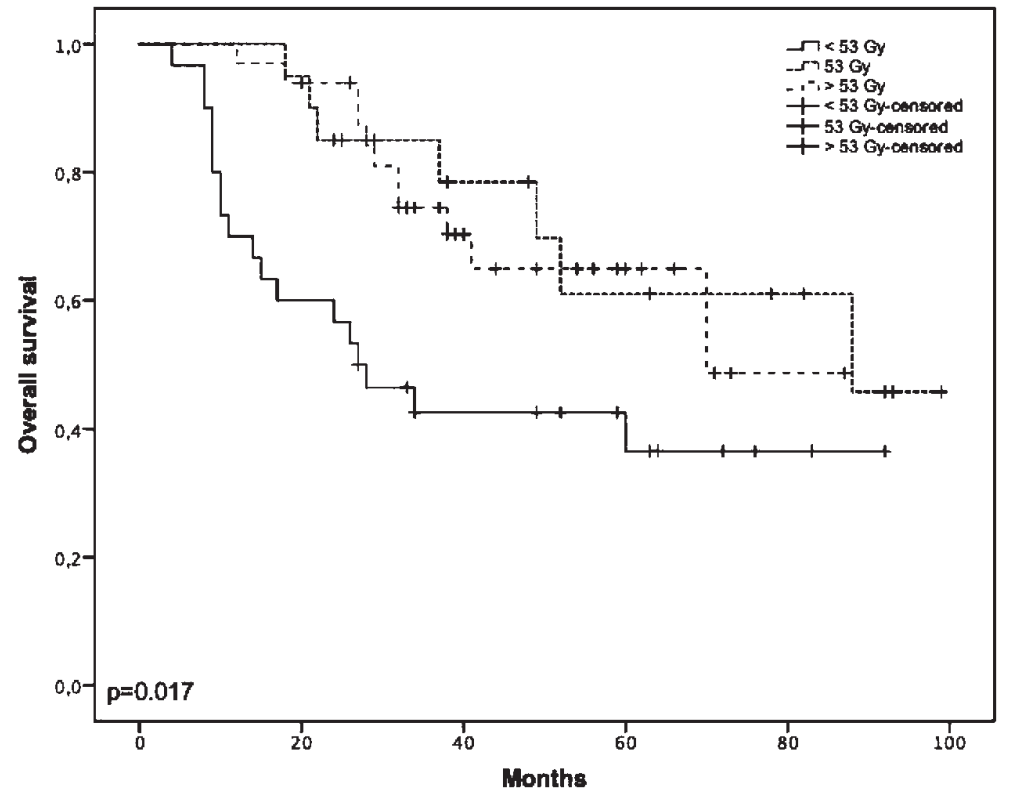

FIGURE 1. Overall survival dependent on parametrial equivalent dose in 2-Gy fractions (EQD2) with $\alpha / \beta=10$.

tic factor for LPFS (T3/4 vs. T1/2, HR $2.668[95 \% \mathrm{CI}$ 1.032-6.896], $\mathrm{p}=0.043)$. Regarding freedom from distant progression (DPFS), presence of pelvic lymph node metastases (N1 vs. N0, HR 4.383 [95\%CI 1.003-19.154], $\mathrm{p}=0.05$ ) as well as distant metastases other than in the paraaortic lymph nodes at initial diagnosis (M1c vs. M0/M1a, HR 4.646 [95\%CI 1.466-14.719], $\mathrm{p}=0.009)$ were independent prognostic factors.

\section{Quality of life analysis}

We found the values of the functioning and symptoms scores in our cohort to be significantly worse comparison those from the normative population (Table 4). Generally, age must be considered when looking at quality of life and we used age as a covariate for our analyses, however age only correlated with menopausal symptoms $(p=0.003)$ in our analysis and did not affect global health status or any of the other functioning or symptom items scores from the QLQ-C30 or QLQ-CX24 questionnaires in our cohort.

The doses to bladder, sigmoid and rectum did not correlate with any of the QoL item scores. However, we found a statistically significant correlation between the degree of observed acute gastrointestinal (GI) and genitourinary (GU) toxicities with chronic symptom experience $(p=0.038$ and $p=0.041$, which can be considered an indicator for the known dose-volume dependence of 
TABLE 3. Prognostic factors for survival

\begin{tabular}{lcc}
\hline & \multicolumn{2}{c}{ Univariate analysis (log-rank test) } \\
\cline { 2 - 3 } OS & $\begin{array}{c}\text { Mean } \\
\text { (months) }\end{array}$ & p-value \\
\hline FIGO stage & 72.6 & 0.015 \\
FIGO I/II & 50.8 & \\
Tumour size & & 0.036 \\
T1/2 & 69.2 & \\
T3/4 & 50.4 & \\
Grading & & 0.053 \\
G1/2 & 72.4 & \\
G3 & 50.5 & 0.037 \\
Parametrial boost & & \\
yes & 69.9 & \\
no & 51.2 & \\
\hline
\end{tabular}

\begin{tabular}{lcc}
\hline \multicolumn{2}{l}{ Parametrial dose in EQD2 $(\alpha / \beta=10)$} & \\
\hline$\leq 50$ Gy & 50.7 & 0.020 \\
$>50$ Gy & 71.5 & \\
$\leq 53$ Gy & 59.5 & 0.194 \\
$>53$ Gy & 64.1 &
\end{tabular}

Chemotherapy

$\begin{array}{lll}\text { yes } & 67.4 & 0.004 \\ \text { no } & 32.1 & \end{array}$

Total treatment duration

$\begin{array}{lcc}6-8 \text { weeks } & 71.6 & 0.027 \\ \geq 9 \text { weeks } & 44.7\end{array}$

Anemia during therapy

$\begin{array}{ll}\min . \mathrm{Hb}<10 \mathrm{~g} / \mathrm{dl} & 52.1 \\ \min . \mathrm{Hb} \geq 10 \mathrm{~g} / \mathrm{dl} & 70.3\end{array}$

$52.1 \quad 0.039$

Transfusions

\begin{tabular}{|c|c|c|}
\hline$\leq 2 \mathrm{ECs}$ & 67.6 & 0.007 \\
\hline$>2 \mathrm{ECs}$ & 41.6 & \\
\hline LPFS & $\begin{array}{c}\text { Mean } \\
\text { (months) }\end{array}$ & p-value \\
\hline \multicolumn{3}{|l|}{ FIGO stage } \\
\hline $\mathrm{FIGO} \mathrm{I/II}$ & 85.9 & 0.072 \\
\hline FIGO III/IV & 66.7 & \\
\hline \multicolumn{3}{|l|}{ Tumour size } \\
\hline $\mathrm{Tl} / 2$ & 85.7 & 0.034 \\
\hline T3/4 & 64.1 & \\
\hline \multicolumn{3}{|c|}{ Parametrial boost } \\
\hline yes & 84.1 & 0.095 \\
\hline no & 56.1 & \\
\hline
\end{tabular}

\begin{tabular}{|c|c|c|c|}
\hline DPFS & $\begin{array}{c}\text { Mean } \\
\text { (months) }\end{array}$ & \multicolumn{2}{|c|}{ p-value } \\
\hline \multicolumn{4}{|c|}{ Lymph node status } \\
\hline NO & 85.6 & \multicolumn{2}{|c|}{0.038} \\
\hline N1 & \multicolumn{3}{|l|}{61.0} \\
\hline \multicolumn{4}{|c|}{ Distant metastases } \\
\hline $\mathrm{MO} / \mathrm{Mla}$ & 75.2 & \multicolumn{2}{|c|}{0.002} \\
\hline Mlc & \multicolumn{3}{|l|}{37.1} \\
\hline \multicolumn{4}{|l|}{ Grading } \\
\hline G1/2 & 84.6 & \multicolumn{2}{|c|}{0.037} \\
\hline G3 & 59.8 & & \\
\hline \multicolumn{4}{|c|}{ Multivariate analysis (Cox regression) } \\
\hline Os & $\mathrm{HR}$ & $95 \% \mathrm{Cl}$ & p-value \\
\hline \multicolumn{4}{|c|}{ Parametrial boost } \\
\hline yes & 0.417 & $0.192-0.900$ & 0.027 \\
\hline no & Reference & & \\
\hline \multicolumn{4}{|c|}{ Chemotherapy } \\
\hline yes & 0.382 & $0.152-0.961$ & 0.041 \\
\hline no & Reference & & \\
\hline LPFS & $\mathrm{HR}$ & $95 \% \mathrm{Cl}$ & p-value \\
\hline \multicolumn{4}{|l|}{ Tumour size } \\
\hline $\mathrm{T} 1 / 2$ & Reference & & \\
\hline $\mathrm{T} 3 / 4$ & 2.668 & $1.032-6.896$ & 0.043 \\
\hline DPFS & HR & $95 \% \mathrm{Cl}$ & p-value \\
\hline \multicolumn{4}{|c|}{ Lymph node status } \\
\hline NO & Reference & & \\
\hline N1 & 4.383 & $1.003-19.154$ & 0.05 \\
\hline \multicolumn{4}{|c|}{ Distant metastases } \\
\hline $\mathrm{MO} / \mathrm{Mla}$ & Reference & & \\
\hline Mlc & 4.646 & $1.466-14.719$ & 0.009 \\
\hline
\end{tabular}

DPFS = Distant progression-free survival; $\mathrm{ECs}=$ erythrocyte concentrates; $\mathrm{EQD2}=$ equivalent doses in 2-Gy fractions; $\mathrm{FIGO}=$ Fédération Internationale de Gynécologie et d'Obstétrique; $\mathrm{Hb}$ = haemoglobin; LPFS = Local progression-free survival OS = overall survival

chronic morbidity. Patients with higher degree of observed acute GI toxicity also complained more about chronic diarrhea $(\mathrm{p}=0.053)$. Since most of our patients underwent IMRT and all of them received HDR ICBT, we found no difference in QoL between RT techniques. Importantly, we could show, that a parametrial EQD2 $10>53$ Gy statistically significantly correlated with reduced sexual/ vaginal functioning $(p=0.009)$ and increased sexual worry $(p=0.009)$. Additionally, these patients also suffered more from chronic constipation $(\mathrm{p}=$ 
0.057). Whether parametrial dose escalation was achieved by sequential boost or SIB did not affect QoL. Sexual/vaginal functioning was statistically significantly worse in patients with T3/4 tumours compared to those with T1/2 tumours $(\mathrm{p}=0.012)$. Details are shown in Table 5.

\section{Discussion}

The objective of this study was to analyze the feasibility and efficacy of a parametrial boost in relation to long-term QoL of patients with ACC. Secondly, we aimed to assess tumour- and treatment-related prognostic factors for long-term QoL and clinical outcome.

Currently, for the QLQ-CX24 items, there are only reference populations from 2 Korean studies available and possible social or cultural differences prevent these cohorts from being reference populations for European studies. ${ }^{10,11}$ Therefore, we were unable to compare the QLQ-CX24 items scores of our patients and we can only report on the comparison between our patients' QLQ-C30 items scores and German normative data. ${ }^{9}$ Compared to the reference population, the patients in our cohort had significantly worse functioning and symptoms item scores. Similar results have been reported in a large population-based study from the United States. They found health-related QoL in survivors of cervical cancer to be worse than in the normal population as well. ${ }^{12}$ In women treated with IGABT within the "European and international study on MRI-guided brachytherapy in locally advanced cervical cancer" (EMBRACE), after a median follow-up of 21 months, functioning and general QoL returned to levels of the reference population, but several clearly treatment-related symptoms, e.g. diarrhea and sexual dysfunction, did develop or persist in those patients during follow-up as well. ${ }^{13}$

While we, probably due to the small cohort, were unable to show a significant correlation between the doses received by the OARs and any of the QoL item scores, others have found a significant dose-volume effect relationship for late rectal and urinary morbidity. Particularly, patients with bladder D2cc > 95 Gy and rectum D2cc $\geq 75$ Gy are at risk for severe late toxicities. ${ }^{14,15}$ It has been shown that chronic bladder and rectal morbidity can be further reduced by IGABT. ${ }^{3}$ Therefore, in the ongoing EMBRACE-2 trial, the planning aims / limits for the prescribed EQD2 3 to rectum (D2cc $<65 /<75$ Gy) and bladder (D2cc $<80 /<90$ Gy) are substantially lower than the mean dose values achieved in
TABLE 4. Quality of life of patients compared to reference populations (EORTC QLQ-C30)

\begin{tabular}{|c|c|c|c|c|}
\hline & $\mathbf{n}$ & mean & SD & $\mathrm{p}$-value \\
\hline \multicolumn{5}{|c|}{ Global health status } \\
\hline Reference & 1309 & 74.5 & 15.5 & 0.001 \\
\hline Patients & 30 & 60.3 & 21.7 & \\
\hline \multicolumn{5}{|c|}{ Physical functioning } \\
\hline Reference & 1309 & 91.5 & 15.5 & $<0.001$ \\
\hline Patients & 30 & 73.6 & 19.6 & \\
\hline \multicolumn{5}{|c|}{ Role functioning } \\
\hline Reference & 1309 & 89.9 & 20.6 & $<0.001$ \\
\hline Patients & 31 & 58.1 & 33.0 & \\
\hline \multicolumn{5}{|c|}{ Emotional functioning } \\
\hline Reference & 1309 & 83.2 & 19.3 & 0.001 \\
\hline Patients & 31 & 65.1 & 26.7 & \\
\hline \multicolumn{5}{|c|}{ Cognitive functioning } \\
\hline Reference & 1309 & 93.4 & 14.6 & $<0.001$ \\
\hline Patients & 31 & 73.2 & 28.4 & \\
\hline \multicolumn{5}{|c|}{ Social functioning } \\
\hline Reference & 1309 & 93.3 & 17.1 & $<0.001$ \\
\hline Patients & 31 & 69.9 & 29.0 & \\
\hline \multicolumn{5}{|l|}{ Fatigue } \\
\hline Reference & 1309 & 16.4 & 21.4 & $<0.001$ \\
\hline Patients & 31 & 51.6 & 28.0 & \\
\hline \multicolumn{5}{|c|}{ Nausea / Vomiting } \\
\hline Reference & 1309 & 2.4 & 9.6 & 0.030 \\
\hline Patients & 31 & 11.3 & 21.7 & \\
\hline \multicolumn{5}{|l|}{ Pain } \\
\hline Reference & 1309 & 17.0 & 24.2 & 0.025 \\
\hline Patients & 31 & 26.9 & 24.2 & \\
\hline \multicolumn{5}{|l|}{ Dyspnea } \\
\hline Reference & 1309 & 7.2 & 18.7 & 0.020 \\
\hline Patients & 30 & 18.9 & 25.8 & \\
\hline \multicolumn{5}{|l|}{ Insomnia } \\
\hline Reference & 1309 & 13.0 & 23.6 & $<0.001$ \\
\hline Patients & 31 & 40.9 & 36.2 & \\
\hline \multicolumn{5}{|l|}{ Appetite loss } \\
\hline Reference & 1309 & 4.2 & 13.9 & 0.002 \\
\hline Patients & 31 & 21.5 & 27.7 & \\
\hline \multicolumn{5}{|l|}{ Constipation } \\
\hline Reference & 1309 & 3.1 & 12.1 & $<0.001$ \\
\hline Patients & 31 & 22.6 & 27.7 & \\
\hline \multicolumn{5}{|l|}{ Diarrhea } \\
\hline Reference & 1309 & 2.9 & 12.7 & $<0.001$ \\
\hline Patients & 31 & 29.0 & 30.7 & \\
\hline \multicolumn{5}{|c|}{ Financial difficulties } \\
\hline Reference & 1309 & 4.8 & 16.3 & $<0.001$ \\
\hline Patients & 31 & 29.0 & 29.5 & \\
\hline
\end{tabular}

EORTC = European Organization for Research and Treatment of Cancer; $Q$ LQ-C30 = quality of life questionnaire for cancer patients 30 
TABLE 5. Prognostic factors for patients' long-term quality of life (EORTC QLQ-C30, QLQ-CX24)

\begin{tabular}{|c|c|c|c|c|}
\hline & $\mathbf{n}$ & mean & SD & p-value \\
\hline \multicolumn{5}{|l|}{ Diarrhea } \\
\hline Acute GI 0 & 11 & 12.1 & 16.8 & 0.053 \\
\hline Acute GI I/II & 15 & 35.6 & 32.0 & \\
\hline Acute GI III/IV & 5 & 46.7 & 38.0 & \\
\hline \multicolumn{5}{|l|}{ Constipation } \\
\hline Parametria $\leq 53 \mathrm{~Gy}^{*}$ & 19 & 14.0 & 23.1 & 0.057 \\
\hline Parametria $>53 \mathrm{~Gy}^{*}$ & 11 & 33.3 & 29.8 & \\
\hline \multicolumn{5}{|l|}{ Symptom experience } \\
\hline Acute $\mathrm{Gl}$ toxicity 0 & 11 & 9.4 & 8.6 & 0.038 \\
\hline Acute GI toxicity I/II & 14 & 20.6 & 15.9 & \\
\hline Acute GI toxicity III/IV & 5 & 30.3 & 22.7 & \\
\hline Acute $\mathrm{GU}$ toxicity 0 & 5 & 14.3 & 12.9 & 0.041 \\
\hline Acute GU toxicity I/II & 22 & 22.4 & 9.7 & \\
\hline Acute GU toxicity III/IV & 3 & 38.4 & 33.5 & \\
\hline \multicolumn{5}{|l|}{ Menopausal symptoms } \\
\hline$\leq 49$ years & 7 & 57.1 & 25.2 & 0.033 \\
\hline $50-59$ years & 12 & 36.1 & 36.1 & \\
\hline $60-69$ years & 7 & 14.3 & 26.2 & \\
\hline$\geq 70$ years & 4 & 8.3 & 16.7 & \\
\hline \multicolumn{5}{|c|}{ Sexual / vaginal functioning } \\
\hline $\mathrm{Tl} / 2$ & 9 & 93.5 & 11.6 & 0.012 \\
\hline $\mathrm{T} 3 / 4$ & 3 & 41.7 & 52.0 & \\
\hline Parametria $\leq 53$ Gy* & 8 & 96.9 & 6.2 & 0.009 \\
\hline Parametria $>53 \mathrm{~Gy}^{*}$ & 4 & 47.9 & 44.2 & \\
\hline \multicolumn{5}{|l|}{ Sexual worry } \\
\hline Parametria $\leq 53 \mathrm{~Gy}^{*}$ & 17 & 15.7 & 26.6 & 0.009 \\
\hline Parametria $>53 \mathrm{~Gy}^{*}$ & 9 & 51.8 & 37.7 & \\
\hline
\end{tabular}

EORTC = European Organization for Research and Treatment of Cancer; GI = gastrointestinal; GU = genitourinary; $Q L Q-C 30$ = quality of life questionnaire for cancer patients 30; QLQ-CX24 = quality of life questionnaire cervical cancer module

*expressed as equivalent dose in 2-Gy fractions (EQD2) with $\mathrm{a} / \mathrm{\beta}=10$

our cohort. ${ }^{5}$ Consequently, the QLQ-CX24 symptom experience score was higher in our patients compared to those treated within the EMBRACE study (18.1 \pm SD 16.4 vs. $12.1 \pm$ SD 11.9; $p=0.063)$ after 36 months of follow-up. ${ }^{13}$ As a further indicator for the dose-volume dependence of chronic morbidity, we found the degree of RT-related acute GI und GU toxicities in patients with ACC to statistically significantly correlate with the extent of chronic symptoms and we have already shown this correlation in a previous study from our institution on women with endometrial cancer. ${ }^{16}$ In that study we also demonstrated an improved global health status and fewer chronic GI symptoms by reduction of acute toxicities with IMRT. ${ }^{16}$ Since most of the patients in our current analysis were treated with IMRT, we were unable to demonstrate such a benefit in terms of long-term QoL. Apart from the dose-volume-relationship found in both, acute and late toxicities, chronic toxicities may be consequential to acute damage, thus amelioration of the acute response to irradiation may be a useful approach to minimize late side effects. ${ }^{17}$

Our analysis showed a significant impairment of sexual/vaginal functioning, significantly increased sexual worry, and a trend towards more chronic constipation in patients exceeding an EQD2 ${ }_{10}$ of $53 \mathrm{~Gy}$ in the parametria. This was equally true for patients with SIB or sequential boost. The required dose to the parametria for adequate local tumour control has not been established and dose prescriptions vary greatly among radiation oncologists.,18 In patients with traditional midline-shielding, parametrial boost resulted in a significantly higher incidence of radiation proctitis and enterocolitis when > 54 Gy were applied. ${ }^{19,20}$ and this technique has been described to substantially contribute to rectum, sigmoid and bladder doses..$^{21}$ In the times of IMRT and SIB to the parametria, studies have shown, that local dose escalation can be achieved with a relevantly reduced incidence of acute toxicities. ${ }^{7,22}$ So far, no study has ever looked at percutaneous parametrial dose escalation regarding QoL and chronic morbidity. Particularly, chronic vaginal morbidity after EBRT boost has not been the focus of research so far. The Vienna group has provided important evidence, that IGABT delivers superior outcome., ${ }^{3,23}$ and can reduce vaginal morbidity significantly, but sexual dysfunction remained a problem in patients treated within the EMBRACE trial as well. ${ }^{13,24}$ It is assumed that vaginal toxicity may be further reduced with IGABT by decreasing dwell times in the ovoid/ring and increasing dwell times in tandem/needles. ${ }^{25,26}$ In this context it is important to state that the ongoing EMBRACE-2 trial limits percutaneous IMRT boost as SIB to involved lymph nodes and does not allow a parametrial IMRT boost. ${ }^{5}$ At our institution, MRI-guided IGABT with combined IBT/ICBT was recently implemented and we are currently planning a study with longitudinal QoL assessment in these patients. Certainly, it should be the ultimate goal to enable access to IGABT at all brachytherapy facilities. Nevertheless, at the moment, availability of IBT and MRI-guidance is low at most brachytherapy centers and EBRT boost remains a widely 
used method for parametrial dose escalation. This underlines the importance of our investigation.

While survival rates were generally adequate in our study and in line with previously published data from randomized controlled trials on RChT in patients with ACC, a comparison of our results to data on IGABT is quite challenging because our cohort consists of mostly advanced stage patients. ${ }^{27,28}$ The RetroEMBRACE study, a multicenter retrospective observational study, found substantially better 5-year pelvic control and overall survival rates ( $84 \%$ vs. $73.2 \%$ and $65 \%$ vs. $53.2 \%)$, but their cohort contained much more stage I and far less stage IV patients than our study $(16.8 \%$ vs. $2.4 \%$ and $3.1 \%$ vs. $31.3 \%$ ) (23). A Dutch retrospective analysis on 46 patients treated with IGABT found a 3-year regional control rate of $86 \%$ and a 3-year overall survival rate of $65 \% .{ }^{29}$ Similarly, Pötter et al. found pelvic control and overall survival to be $91 \%$ and $68 \%$ after 3 -years, respectively. ${ }^{3}$ The patient cohorts in these two studies were very similar to the collective of the RetroEMBRACE study. Therefore, our results, despite the large proportion of stage IV patients and the negligible amount of stage I patients, are not inferior in terms of overall survival and seem comparable with respect to local pelvic control. However, primary tumour control was remarkably high with $93 \%$ after 3 years in the Vienna series. ${ }^{3}$ Furthermore, an analysis from the RetroEMBRACE study comparing IGABT with IBT/ICBT to ICBT only showed the feasibility of local dose escalation without an increase in dose to organs at risk as well as a $10 \%$ higher 3-year local control rate with IBT. ${ }^{30}$ These results clearly demonstrate the advantage of MRI-based IGABT with combined IBT/ICBT for local dose escalation.

Nevertheless, our analysis showed a statistically significant overall survival benefit from a parametrial boost and this was equally true for patients with SIB or sequential boost. Alongside with simultaneous ChT, parametrial boost was as an independent prognostic factor for OS in the multivariate analysis. Local control was also improved in patients with parametrial boost, but, probably due to the comparatively few events, this did not reach statistical significance. However, the survival benefit was dependent on the applied dose. In particular, overall survival was statistically significantly prolonged in patients with a parametrial EQD2 ${ }_{10}>$ $50 \mathrm{~Gy}$, but a dose escalation beyond an EQD2 ${ }_{10}$ of 53 Gy did not further improve survival. Thus, we believe IMRT with $25 \times 1.8$ Gy to the whole pelvic PTV including a SIB with $25 \times 2.1$ Gy to the para- metria to be a feasible and overall efficient EBRT concept for centers without access to IGABT.

We acknowledge that our study is substantially limited by its retrospective nature, the comparatively small sample size and the conductance of multiple subgroup analyses, but we believe our results on parametrial dose and chronic morbidity to be of importance for all brachytherapy centers without access to MRI-guidance and IBT.

Primary $\mathrm{RChT}$ is an effective treatment for ACC, but long-term QoL of survivors is inferior compared to normative data. The degree of acute side effects of RChT correlates with the extent of chronic symptoms. For patients treated with EBRT boost and ICBT, we have shown a significant survival benefit from parametrial dose escalation as SIB or sequential boost, but exceeding an EQD2 ${ }_{10}$ of 53 Gy with this technique does not further improve survival and has a negative impact on QoL. Therefore, the conductance of a percutaneous parametrial boost has to be seen very critically with respect to local tumour control and long-term QoL. MRI-guided IGABT with combined IBT/ICBT certainly is the gold standard for local dose escalation in ACC.

\section{References}

1. Haie-Meder C, Potter R, Van Limbergen E, Briot E, De Brabandere M Dimopoulos J, et al. Recommendations from Gynaecological (GYN) GECESTRO Working Group (I): concepts and terms in 3D image based 3D treatment planning in cervix cancer brachytherapy with emphasis on MRI assessment of GTV and CTV. Radiother Oncol 2005; 74: 235-45. doi: 10.1016/j.radonc.2004.12.015

2. Viswanathan $A N$, Creutzberg $C L$, Craighead P, McCormack M, Toita $T$, Narayan K, et al. International brachytherapy practice patterns: a survey of the Gynecologic Cancer Intergroup (GCIG). Int J Radiat Oncol Biol Phys 2012; 82: 250-5. doi: $10.1016 /$ j.jirobp.2010.10.030

3. Potter R, Georg P, Dimopoulos JC, Grimm M, Berger D, Nesvacil N, et al. Clinical outcome of protocol based image (MRI) guided adaptive brachytherapy combined with 3D conformal radiotherapy with or without chemotherapy in patients with locally advanced cervical cancer. Radiother Oncol 2011; 100: 116-23. doi: 10.1016/j.radonc.2011.07.012

4. Rijkmans EC, Nout RA, Rutten IH, Ketelaars M, Neelis KJ, Laman MS, et al. Improved survival of patients with cervical cancer treated with imageguided brachytherapy compared with conventional brachytherapy. Gynecol Oncol 2014; 135: 231-8. doi: 10.1016/j.ygyno.2014.08.027

5. Potter R, Tanderup K, Kirisits C, de Leeuw A, Kirchheiner K, Nout R, et al. The EMBRACE II study: the outcome and prospect of two decades of evolution within the GEC-ESTRO GYN working group and the EMBRACE studies. Clin Transl Radiat Oncol 2018; 9: 48-60. doi: 10.1016/j.ctro.2018.01.001

6. Mohamed S, Kallehauge J, Fokdal L, Lindegaard JC, Tanderup K. Parametrial boosting in locally advanced cervical cancer: combined intracavitary/interstitial brachytherapy vs. intracavitary brachytherapy plus external beam radiotherapy. Brachytherapy 2015; 14: 23-8. doi: 10.1016/j.brachy.2014.09.010

7. Marnitz S, Kohler C, Burova E, Wlodarczyk W, Jahn U, Grun A, et al. Helical tomotherapy with simultaneous integrated boost after laparoscopic staging in patients with cervical cancer: analysis of feasibility and early toxicity. Int $J$ Radiat Oncol Biol Phys 2012; 82: e137-43. doi: 10.1016/j.jirobp.2010.10.066 
8. Potter R, Haie-Meder C, Van Limbergen E, Barillot I, De Brabandere M, Dimopoulos J, et al. Recommendations from gynaecological (GYN) GEC ESTRO working group (II): concepts and terms in 3D image-based treatment planning in cervix cancer brachytherapy-3D dose volume parameters and aspects of 3D image-based anatomy, radiation physics, radiobiology. Radiother Oncol 2006; 78: 67-77. doi: 10.1016/j.radonc.2005.11.014

9. Hinz A, Singer S, Brahler E. European reference values for the quality of life questionnaire EORTC QLQ-C30: results of a German investigation and a summarizing analysis of six European general population normative studies. Acta Oncol 2014; 53: 958-65. doi: 10.3109/0284186x.2013.879998

10. Lee Y, Lim MC, Kim SI, Joo J, Lee DO, Park SY. Comparison of quality of life and sexuality between cervical cancer survivors and healthy women. Cancer Res Treat 2016; 48: 1321-9. doi: 10.4143/crt.2015.425

11. Park SY, Bae DS, Nam JH, Park CT, Cho CH, Lee JM, et al. Quality of life and sexual problems in disease-free survivors of cervical cancer compared with the general population. Cancer 2007; 110: 2716-25. doi: 10.1002/ cncr.23094

12. Weaver KE, Forsythe LP, Reeve BB, Alfano CM, Rodriguez JL, Sabatino SA, et al. Mental and physical health-related quality of life among U.S. cancer survivors: population estimates from the 2010 National Health Interview Survey. Cancer Epidemiol Biomarkers Pre 2012; 21: 2108-17. doi: 10.1158/1055-9965.EPI-12-0740

13. Kirchheiner K, Potter R, Tanderup K, Lindegaard JC, Haie-Meder C, Petric $P$, et al. Health-related quality of life in locally advanced cervical cance patients after definitive chemoradiation therapy including image guided adaptive brachytherapy: an analysis from the EMBRACE study. Int J Radiat Oncol Biol Phys 2016; 94: 1088-98. doi: 10.1016/j.jirobp.2015.12.363

14. Kim Y, Kim YJ, Kim JY, Lim YK, Jeong C, Jeong J, et al. Toxicities and dosevolume histogram parameters of MRI-based brachytherapy for cervical cancer. Brachytherapy 2017; 16: 116-25. doi: 10.1016/j.brachy.2016.10.005

15. Mazeron R, Maroun P, Castelnau-Marchand P, Dumas I, del Campo ER, Cao $\mathrm{K}$, et al. Pulsed-dose rate image-guided adaptive brachytherapy in cervical cancer: dose-volume effect relationships for the rectum and bladder. Radiother Oncol 2015; 116: 226-32. doi: 10.1016/j.radonc.2015.06.027

16. Foerster $\mathrm{R}$, Schnetzke L, Bruckner $\mathrm{T}$, Arians $\mathrm{N}$, Rief $\mathrm{H}$, Debus J, et al. Prognostic factors for long-term quality of life after adjuvant radiotherapy in women with endometrial cancer. Strahlenther Onkol 2016; 192: 895-904. doi: 10.1007/s00066-016-1037-1

17. Dorr W, Hendry JH. Consequential late effects in normal tissues. Radiother Oncol 2001; 61: 223-31. doi: 10.1016/S0167-8140(01)00429-7

18. Rajasooriyar C, Van Dyk S, Lindawati M, Bernshaw D, KondalsamyChennakesavan S, Narayan K. Reviewing the role of parametrial boost in patients with cervical cancer with clinically involved parametria and staged with positron emission tomography. Int J Gynecol Cancer 2012; 22: 1532-7. doi: 10.1097/IGC.0b013e31826c4dee

19. Huang EY, Lin $\mathrm{H}$, Hsu HC, Wang CJ, Chen HC, Sun LM, et al. High externa parametrial dose can increase the probability of radiation proctitis in patients with uterine cervix cancer. Gynecol Oncol 2000; 79: 406-10. doi: 10.1006/gyno.2000.5997

20. Huang EY, Wang CJ, Hsu HC, Hao L, Chen HC, Sun LM. Dosimetric factors predicting severe radiation-induced bowel complications in patients with cervical cancer: combined effect of external parametrial dose and cumulative rectal dose. Gynecol Oncol 2004; 95: 101-8. doi: 10.1016/j. ygyno.2004.06.043

21. Fenkell L, Assenholt M, Nielsen SK, Haie-Meder C, Potter R, Lindegaard J, et al. Parametrial boost using midline shielding results in an unpredictable dose to tumor and organs at risk in combined external beam radiotherapy and brachytherapy for locally advanced cervical cancer. Int J Radiat Oncol Biol Phys 2011; 79: 1572-9. doi: 10.1016/j.jirobp.2010.05.031

22. Boyle J, Craciunescu O, Steffey B, Cai J, Chino J. Methods, safety, and early clinical outcomes of dose escalation using simultaneous integrated and sequential boosts in patients with locally advanced gynecologic malignancies. Gynecol Oncol 2014; 135: 239-43. doi: 10.1016/j.ygyno.2014.08.037

23. Sturdza A, Potter R, Fokdal LU, Haie-Meder C, Tan LT, Mazeron R, et al. Image guided brachytherapy in locally advanced cervical cancer: improved pelvic control and survival in RetroEMBRACE, a multicenter cohort study. Radiother Oncol 2016; 120: 428-33. doi: 10.1016/j.radonc.2016.03.011
24. Kirchheiner K, Nout RA, Tanderup K, Lindegaard JC, Westerveld H, HaieMeder $\mathrm{C}$, et al. Manifestation pattern of early-late vaginal morbidity after definitive radiation (chemo)therapy and image-guided adaptive brachytherapy for locally advanced cervical cancer: an analysis from the EMBRACE study. Int J Radiat Oncol Biol Phys 2014; 89: 88-95. doi: 10.1016/j. ijrobp.2014.01.032

25. Kirchheiner K, Nout RA, Lindegaard JC, Haie-Meder C, Mahantshetty U, Segedin B, et al. Dose-effect relationship and risk factors for vaginal stenosis after definitive radio(chemo)therapy with image-guided brachytherapy for locally advanced cervical cancer in the EMBRACE study. Radiother Oncol 2016; 118: 160-6. doi: 10.1016/j.radonc.2015.12.025

26. Mohamed S, Lindegaard JC, de Leeuw AA, Jurgenliemk-Schulz I, Kirchheiner $\mathrm{K}$, Kirisits $\mathrm{C}$, et al. Vaginal dose de-escalation in image guided adaptive brachytherapy for locally advanced cervical cancer. Radiother Oncol 2016; 120: 480-5. doi: 10.1016/j.radonc.2016.05.020

27. Morris M, Eifel PJ, Lu J, Grigsby PW, Levenback C, Stevens RE, et al. Pelvic radiation with concurrent chemotherapy compared with pelvic and paraaortic radiation for high-risk cervical cancer. N Engl J Med 1999; 340: 113743. doi: 10.1056/nejm199904153401501

28. Rose PG, Bundy BN, Watkins EB, Thigpen JT, Deppe G, Maiman MA, et al. Concurrent cisplatin-based radiotherapy and chemotherapy for locally advanced cervical cancer. N Engl J Med 1999; 340: 1144-53. doi: 10.1056/ nejm199904153401502

29. Nomden CN, de Leeuw AA, Roesink JM, Tersteeg RJ, Moerland MA, Witteveen PO, et al. Clinical outcome and dosimetric parameters of chemoradiation including MRI guided adaptive brachytherapy with tandem-ovoid applicators for cervical cancer patients: a single institution experience. Radiother Oncol 2013; 107: 69-74. doi: 10.1016/j.radonc.2013.04.006

30. Fokdal L, Sturdza A, Mazeron R, Haie-Meder C, Tan LT, Gillham C, et al. Image guided adaptive brachytherapy with combined intracavitary and interstitial technique improves the therapeutic ratio in locally advanced cervical cancer: Analysis from the retroEMBRACE study. Radiother Oncol 2016; 120 434-40. doi: 10.1016/j.radonc.2016.03.020 